\title{
Development of an acrylamide-based inhibitor of protein S-acylation
}

Saara-Anne Azizi ${ }^{1,2, \#}$, Tong Lan ${ }^{1, \#}$, Clémence Delalande ${ }^{1}$, Rahul S. Kathayat ${ }^{1}$, Fernando Banales Mejia ${ }^{1}$, Alice Qin ${ }^{1}$, Noah Brookes ${ }^{1}$, Perla Jasmine Sandoval ${ }^{1}$, Bryan C. Dickinson ${ }^{1}$, *

${ }^{1}$ Department of Chemistry, The University of Chicago, Chicago, Illinois 60637, United States

${ }^{2}$ Medical Scientist Training Program, Pritzker School of Medicine, The University of Chicago, Chicago, Illinois 60637, United States

*Dickinson@uchicago.edu

\#These authors contributed equally to this work.

\begin{abstract}
Protein S-acylation is a dynamic lipid post-translational modification that can modulate the localization and activity of target proteins. In humans, the installation of the lipid onto target proteins is catalyzed by a family of 23 Asp-His-His-Cys domain-containing protein acyltransferases (DHHC-PATs). DHHCs are increasingly recognized as critical players in cellular signaling events and in human disease. However, progress elucidating the functions and mechanisms of DHHC "writers" has been hampered by a lack of chemical tools to perturb their activity in live cells. Herein, we report the synthesis and characterization of cyanomyracrylamide (CMA), a broad-spectrum DHHC family inhibitor with similar potency to 2bromopalmitate (2BP), the most commonly used DHHC inhibitor in the field. Possessing an acrylamide warhead instead of 2BP's a-halo fatty acid, CMA inhibits DHHC family proteins in cellulo while demonstrating decreased toxicity and avoiding inhibition of the $S$-acylation eraser enzymes - two of the major weaknesses of 2BP. Our studies show that CMA engages with DHHC family proteins in cells, inhibits protein S-acylation, and disrupts DHHC-regulated cellular events.
\end{abstract} CMA represents an improved chemical scaffold for untangling the complexities of DHHCmediated cell signaling by protein S-acylation. 


\section{Introduction}

Protein S-acylation is the post-translational addition of a long chain fatty acid to cysteine thiols via a thioester bond ${ }^{1-2}$ and is often referred to as $S$-palmitoylation due to the prevalence of C16:0 modification. S-acylation has complex and wide-ranging effects on target proteins; not only can it alter membrane association and subcellular trafficking, as for the well-studied oncogene Ras $^{3}$, but it also regulates protein oligomerization, activity, and stability ${ }^{3-7}$. S-acylation is enzymatically reversible, with lipid addition catalyzed by the protein acyl transferases (PATs), which possess an active site Asp-His-His-Cys domain (earning them the moniker "DHHC") and lipid removal, by a suite of serine hydrolase family acyl-protein thioesterases (APTs), including APT1 ${ }^{8}, \mathrm{APT}^{9}$, $\mathrm{ABHD} 10^{6}$, and $\mathrm{ABHD} 17 \mathrm{~A} / \mathrm{B} / \mathrm{C}^{10}$. The activity of both these DHHC "writer" and APT "eraser" proteins is tightly regulated, and together, they in turn dynamically regulate protein $S$-acylation. Disruption of this cycle is consequential at the cellular and organismal levels ${ }^{11-13}$.

In particular, dysregulation of DHHC family proteins is associated with human pathology, including cancer progression, inflammation, and neurological dysfunction ${ }^{14-16}$. For example, zDHHC9 is upregulated in colorectal cancer and has been implicated in the pathogenesis of leukemia ${ }^{17-18}$, while its loss-of-function mutations are associated with X-linked intellectual disability (XLID) ${ }^{19-20}$. $\mathrm{ZDHHC20}$ regulates the signaling of the receptor tyrosine kinase EGFR, and its activity has been implicated in cellular transformation and lung tumorigenesis ${ }^{21-22}$. Finally, knockdown of zDHHC7 has been shown to mitigate symptoms of inflammatory bowel disease, possibly via loss of STAT3 palmitoylation and activation ${ }^{23}$.

Despite the annotation of the first palmitoyl transferase in yeast two decades ago ${ }^{24-25}$, a paucity of tools to perturb the function of the $\mathrm{DHHC}$ family has hindered progress in our understanding of their mechanism, regulatory roles, and connections to disease states. Functional redundancy between many DHHCs, as well as inter-family regulation ${ }^{26-28}$, limit the use of classic genetic tools and highlight the necessity of small molecule inhibitors. While the a-brominated fatty acid 2bromopalmitate (2BP) is the most commonly used small molecule tool to study DHHC functions in live cells, its frequent use obscures its promiscuity, poor utility, and cellular toxicity. 2BP is hypothesized to act as a pan-DHHC inhibitor by covalently modifying the active cysteine residues of $\mathrm{DHHC}$ proteins, either as $2 \mathrm{BP}$ or the 2BP-CoA adduct, with the latter metabolic product displaying enhanced reactivity with proteome ${ }^{29}$. In fact, 2BP inhibits at least two of the S-acylation erasers, APT1 and APT2, in cells ${ }^{30}$. Moreover, 2BP is often used at micromolar concentrations $(10-100 \mu \mathrm{M})$, concentrations that are at or above its toxicity threshold ${ }^{27,29,31}$. Other reported $S$ - 
acylation inhibitors, such as cerulenin, tunicamycin, and compound $\mathrm{V}$, suffer from significant toxicity, poor selectively, and/or a lack of characterization in cells ${ }^{32-34}$. Thus, there is a critical need for new chemical probes to study DHHC-mediated S-acylation in endogenous contexts.

Here, we report the development of CMA, a covalent broad-spectrum DHHC inhibitor with an acrylamide warhead and improved properties relative to 2BP. Not only is CMA more potent than 2BP in vitro, it is also less toxic and does not inhibit APT1 or APT2, thereby addressing two key limitations of 2BP. We demonstrate that CMA inhibits S-acylation and engages directly with DHHC family proteins in cellulo. Finally, we show that CMA can modulate DHHC-dependent cellular effects, including EGFR-mediated cell signaling and CD36-mediated lipid uptake and droplet formation. Overall, this work introduces CMA as a chemical tool that inhibits a broad range of DHHCs and complements current methods to study the biological implications of disrupting DHHC-mediated S-acylation.

\section{Acrylamide-based molecules inhibit zDHHC20}

The DHHC family proteins are thought to employ a two-step mechanism, in which the cysteine thiolate of the active site attacks the fatty acyl-CoA thioester, resulting in an autoacylated DHHC with the acyl chain bound in the hydrophobic groove. The fatty acyl chain is then transferred from the cysteine to a protein substrate. This nucleophilic cysteine can be targeted by electrophiles like 2BP - to hamper DHHC activity. In pursuit of a still potent but less reactive inhibitor in cellulo, we proposed to exchange the $\alpha$-halo carbonyl of 2BP with an acrylamide warhead. Acrylamides are known to react faster with cysteine thiols than serine alcohols, and, unlike the 2BP fatty acid, will likely not undergo metabolic conversion to reactive and nonspecific acyl CoA intermediates 29, 35. Moreover, the acrylamide scaffold is achiral and readily synthetically accessible. Therefore, we designed and synthesized 1 (Figure 1A), which features a 14 carbon-long lipid tail appended to the acrylamide warhead. Docking of 1 on the only published crystal structure of a human DHHC (zDHHC20) showed that the warhead is proximal (3.5 $\AA$ ) to the active site Cys (Figure S1). We therefore tested 1 against purified $\mathrm{zDHHC} 20$ using a fluorescence polarization (FP) assay adapted from Acyl-cLIP and using a fluorophore-tagged peptide that we identified as a substrate of zDHHC20, 5-FAM-NRas (Figure S2) ${ }^{36} .1\left(_{50}=21.4 \pm 5.9 \mu \mathrm{M}\right)$ successfully inhibited zDHHC20, although with decreased activity as compared to $2 \mathrm{BP}\left(\mathrm{IC}_{50}=5.33 \pm 0.77 \mu \mathrm{M}\right)$, confirming that an acrylamide-based lipid inhibitor can inhibit zDHHC20. Thus, we decided to explore the structure-activity relationship of 1 in an effort to enhance its potency. 


\section{CMA is a potent zDHHC20 inhibitor}

To optimize 1, we designed and synthesized a panel of compounds, all with an acrylamide appended to a lipid tail. First, we targeted the lipid tail, shortening it by four carbons (2). This change abrogated the zDHHC20 inhibitory properties entirely, demonstrating the criticality of extensive contacts with the hydrophobic channel and inspiring us to instead focus on modifying the acrylamide moiety. We next tested compounds with either a substituted acrylamide amine and/or a butenoic acid-modified amide (Figure 1B). While most performed worse than the parent molecule, one molecule functionalized with a cyanomethyl group, CMA, showed significantly improved activity against zDHHC20, with approximately $80 \%$ inhibition at $10 \mu \mathrm{M}$. CMA was found to have an $\mathrm{IC}_{50}$ of $1.35 \pm 0.26 \mu \mathrm{M}$ against $\mathrm{zDHHC} 20$ with 1 hour of preincubation, a 5-fold improvement over 2BP (Figure S3). We further characterized the kinetic parameters of covalent modification, $\mathrm{K}_{\text {inact }}$ and $\mathrm{K}_{\mathrm{I}}$ for both CMA and 2BP, observing that CMA had both a faster reaction rate $\left(2.08\right.$ vs. $\left.1.35 \mathrm{~min}^{-1}\right)$ and a stronger affinity towards $\mathrm{zDHHC} 20(5.8$ vs. $6.4 \mu \mathrm{M})$ than $2 \mathrm{BP}$ (Figure S4). Moreover, addition of the cyanomethyl group to the cis butenoic acid derivative (3) improved its activity (12), highlighting the criticality of this moiety. A second molecule, $\mathbf{8}$, which possesses a terminal alkyne in lieu of the cyanomethyl, was similar in potency to 2BP, with an $\mathrm{IC}_{50}$ of $8.32 \pm 2.25 \mu \mathrm{M}$ (Figure S3). To confirm that the reactivity of CMA and 8 stemmed from the acrylamide warhead and not reversible thioimidate complex formation, we synthesized and tested 13 and 14, the alkyl amide analogues of 8 and CMA, respectively (Figure 1B). Both molecules were inactive, verifying the acrylamide as the DHHC-reactive moiety. In sum, CMA is a potent zDHHC20 inhibitor in vitro and requires both the cyanomethyl group and acrylamide warhead for activity. Therefore, to assess its utility in live cells, we next assessed its inhibition of APTs and toxicity.

\section{CMA does not inhibit APTs and is less toxic than 2BP}

To determine if CMA inhibits eraser APTs, as observed with 2BP, we evaluated the effects of CMA relative to 2BP on APT1 and APT2 activity in vitro using a fluorogenic probe for APT activity, DPP5 ${ }^{37}$. CMA showed little inhibition of APT1 and APT2, while 2BP, in agreement with previous reports, significantly inhibited both APT1 and APT2 (Figure $2 \mathrm{~A})^{30}$. At $25 \mu \mathrm{M}$, a concentration at or below which 2BP is often used, 2BP abolished most activity of APT1 and all activity of APT2. In striking contrast, CMA showed no inhibition of APT1 or APT2 even at $50 \mu \mathrm{M}$, the highest concentration assayed. This data suggests that CMA, unlike 2BP, can be used to monitor the dynamics and the kinetics of acylation without perturbing APT-mediated deacylation. 
We also compared the toxicity of CMA to that of 2BP across a panel of commonly used mammalian cell lines, including MDA-MB-231, HEK293T, and 3T3-L1. After 24 hours of treatment, CMA's toxicity was limited up to $40 \mu \mathrm{M}$, while 2BP significantly reduced cell viability at much lower concentrations (Figure 2B, S5). For example, in MDA-MB-231 cells, treatment with 2BP resulted in $\sim 90 \%$ cell death at $20 \mu \mathrm{M}$, whereas at the same concentration of CMA, no toxicity was observed. However, as the toxicity of both compounds was cell-line dependent, CMA treatment time and concentration will need to be optimized for a particular experimental system. As CMA was non-toxic at $20 \mu \mathrm{M}$ across all cell lines we tested, we designated this as our maximum concentration of CMA to use for assessment in these cell lines. Overall, these results - the lack of APT inhibition and decreased toxicity - paired with the robust inhibition of zDHHC20 in vitro, suggested that CMA might be an inhibitor of zDHHC-mediated acylation in cells.

\section{CMA inhibits protein S-acylation in live cells}

To validate the use of CMA as an inhibitor of S-acylation, we next sought to assess its efficacy and potency in live cells. Using 17-octadecynoic acid (17-ODYA) metabolic labeling, wherein a clickable fatty acid is used to monitor lipid incorporation, we observed a dose-dependent decrease in global S-acylation when cells were treated with CMA (Figure 3A, S6) ${ }^{38}$. We further confirmed the global inhibition of protein S-acylation by CMA using acyl-biotin exchange (ABE) $)^{39}$, a method for the enrichment and visualization of acylated cysteine residues (Figure 3B, S7).

To corroborate these proteome-wide observations and also to evaluate the conditions of CMA inhibition, we next tested whether and when CMA could inhibit the S-acylation of several wellstudied DHHC substrate proteins. We first assessed its effect on the S-acylation status of overexpressed Legionella E3 ligase GobX, the overexpressed human immune adaptor protein Myd88, and the endogenous GTPase Ras, which are regulated by zDHHC20, zDHHC9, and zDHHC6, respectively ${ }^{40-41}$. After treating HEK293T cells with a range of concentrations of CMA under serum-free conditions, we observed a dose-dependent decrease in the S-acylation of overexpressed GobX and Myd88 and endogenous Ras, with a significant loss of acylation at 20 $\mu \mathrm{M}$ after 6 hours of treatment for all three substrates (Figure $3 \mathrm{C}$ ). We then assessed the time course of CMA inhibition of these two targets at $0,1,3$, and 6 hours, observing significant inhibition for these particular substrates only after 6 hours of treatment (Figure S8). However, as the turnover of acylation is substrate-dependent, the time component of CMA inhibition will need to be optimized for individual substrates ${ }^{42-43}$. Additionally, as the non-specific binding with serum proteins in culture media is a known challenge for small molecule inhibitors, we also assayed the 
effect of serum concentration on CMA alongside 2BP, probing both global and GobX S-acylation. Using $\mathrm{ABE}$, we observe that in up to 1\% FBS, both CMA and 2BP inhibit global and GobX Sacylation, but with 10\% FBS, the activity of both molecules appears extremely limited (Figure 3D, S9). Overall, all targets displayed reduced acylation at a concentration of $20 \mu \mathrm{M}$ CMA with 6 hours of treatment, establishing this as our working concentration. Collectively, these data confirm that CMA inhibits protein S-acylation in live cells.

\section{CMA engages DHHCs in live cells}

Having verified that CMA inhibits zDHHC20 in vitro and blocks protein S-acylation in live cells, we next sought to confirm direct engagement of DHHC family proteins by CMA in live cells. We postulated that 8, an analogue of CMA possessing a terminal alkyne instead of the cyanomethyl group, could be used to visualize CMA engagement across the DHHC family by reporter conjugation via copper-catalyzed azide-alkyne "click" cycloaddition (Figure 1A). Therefore, we first visualized 8 labeling across the proteome, subjecting 8-treated HEK293T cell lysates (10 $\mu \mathrm{M}, 2$ hours) to the click reaction with TAMRA-azide. In-gel fluorescence indicated labeling in both serum-free and 10\% FBS-complemented media, although labeling rate and intensity were decreased under the latter conditions (Figure S10). We next determined which DHHCs could be labeled by $\mathbf{8}$. HEK293T cells overexpressing individual DHHCs were treated with 8 (10 $\mu \mathrm{M}, 2$ hours), and, as above, following cell lysis, subjected to a click reaction with TAMRA-azide in order to label 8-modified proteins. Analysis of in-gel fluorescence showed that 8 labels zDHHC2, 4, 6, 9, 11, 13, 14, 15, 16, 18, 20, 23, and 24, all of the DHHCs in our human and murine libraries with strong expression (Figure S10A-C, 11). Furthermore, 8 was also found to label endogenous DHHC proteins, including ZDHHC5 and 7, which was not labeled in the fluorescence screen, likely due to poor expression (Figure S12). Notably, the labeling intensity and range of 8 differed from 16C-BYA, possibly suggesting different selectivity profiles for CMA and 2BP.

We next examined the residues of DHHC proteins labelled by $\mathbf{8}$. As the cysteine residue within the DHHC motif is critical for the S-acyltransferase activity for DHHC proteins ${ }^{24-25,44-45}$, we performed 8 labeling on a subset of exogenous catalytically dead (DHHS) mutants. While some DHHCs, such as zDHHC9 and 24, evinced decreased labeling of the active site mutant, other DHHCs, including zDHHC20, did not, suggesting that the DHHC motif Cys is not the only residue modified by $\mathbf{8}$, as is also observed with 16C-BYA, an alkyne-containing analogue of $2 \mathrm{BP}^{46}$ (Figure S13). While the functional consequences of the 2BP and CMA reactivity with peripheral cysteines 
remain unknown, this result could suggest a possible role for these residues in the mechanism of inhibition.

The background fluorescence observed during in-gel labeling with 8 suggested that CMA has offtargets in cells. Thus, we decided to use mass spectrometry (MS) to profile the proteome-wide reactivity of $\mathbf{8}$ and therefore CMA. To this end, following treatment of HEK293T cells with $20 \mu \mathrm{M}$ 8 or DMSO, click reaction with biotin-azide, enrichment, and on-bead trypsin digestion, digested peptides were identified and quantified by dimethyl labeling. Consistent with the gel-based results, we found that 8 had a wide range of off-targets in cells (Figure S15, Table S1). When filtered with $\geq 2$ unique peptides and $\geq 5$-fold enrichment in at least 2 of 3 replicates, 271 targets can be identified. Top enriched proteins include chitobiosyldiphosphodolichol beta-mannosyltransferase (ALG1), threonylcarbamoyl adenosine tRNA methylthiotransferase (CDKAL1), and neutral cholesterol ester hydrolase 1 (NCEH1), while off-targets (targets other than DHHC family members) more broadly included transferases, translocases, and channel proteins. Unsurprisingly, due to the low abundance and have high hydrophobicity of DHHC family proteins, LC-MS/MS analysis only identified one DHHC protein (zDHHC6), which did not even pass the

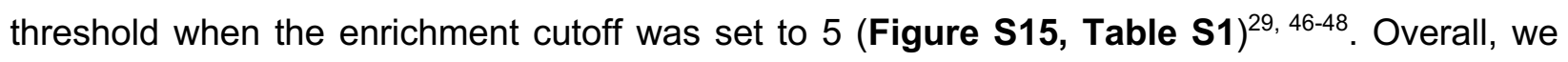
hope that this proteomics data can provide a guide for optimizing future derivatives of CMA against off-targets.

Having profiled the reactivity of $\mathbf{8}$ and validated its ability to label DHHC family proteins, we next performed competitive labeling with CMA for exogenous (zDHHC2, zDHHC9, zDHHC14, zDHHC15, and zDHHC24) and endogenous (zDHHC5, 13, and 18) DHHC proteins labeled by 8 in order to confirm CMA target engagement in live cells. For the exogenous DHHCs, cells overexpressing each targeted protein were treated with CMA $(0,10$, and $20 \mu \mathrm{M}, 2$ hours) in either serum-free or 10\% FBS media, chased with 8 ( $1 \mu \mathrm{M}, 2$ hours), and then lysed. After TAMRAazide ligation, in-gel fluorescence indicated that CMA blocked 8 labeling, confirming that CMA engages each of these DHHC targets (Figure 4A, S10D, S14). To probe the endogenous DHHCs, we again performed competitive labeling, this time on non-transfected HEK293T cells and using 8 at $20 \mu \mathrm{M}$ for 3 hours for the chase. Here, after conjugation with biotin-azide, pulldown, and Western blotting, we observed that CMA impeded the 8 labeling of $\mathrm{ZDHHC5}, \mathrm{zDHHC} 18$, and two isoforms of zDHHC13 (Figure 4B) - confirming CMA directly engages with these DHHCs as well. The ability of CMA to outcompete 8 labeling of both exogenous and endogenous DHHCs 
suggests that CMA inhibition of $S$-acylation in live cells likely stems from inhibition of DHHC family proteins.

\section{CMA inhibits the S-acylation of EGFR and CD36}

Finally, we sought to test whether CMA could recapitulate observations reported to stem from DHHC disruption and establish that, along with on-target binding, CMA can be used to discern cellular outcomes from loss of S-acylation. A critical S-acylated protein and substrate of zDHHC20 is the epidermal growth factor receptor (EGFR), a receptor tyrosine kinase whose activity is widely dysregulated in cancer ${ }^{21}$. There are several mechanisms by which its C-terminal acylation regulates its activity; in a KRAS-mutant context, loss of its palmitoylation is reported to disrupt signaling along the phosphatidylinositol 3-kinase (PI3K) pathway ${ }^{22}$. Thus, we first confirmed that CMA diminished EGFR S-acylation in MDA-MB-231 cancer cells at its working concentration, but that 14, the non-covalent analogue of CMA did not (Figure 5A, S16). We then assessed whether CMA-mediated interruption of EGFR acylation impacted downstream PI3K pathway signaling. We observed that treatment with CMA, but not with 14, moderated the phosphorylation of protein kinase B (Akt) and the abundance of the transcription factor MYC, two markers of EGFR-P13K pathway activity (Figure 5B, S17). Interestingly, treatment with 10, a molecule inactive against zDHHC20 in vitro, inhibited EGFR acylation and PI3K pathway activation in cellulo, possibly implicating another $\mathrm{ZDHHC}$ in the regulation of the EGFR pathway (Figure S16, 17C). Overall, these results paralleled those seen with genetic knockdown of zDHHC20 and established CMA's ability to validate results downstream of acylation disruption ${ }^{22}$.

To further substantiate CMA's ability to modulate DHHC-dependent cellular functions, we next evaluated whether CMA could inhibit the $S$-acylation and subsequently the activity of CD36. CD36 is an immuno-metabolic glycoprotein and a substrate of zDHHC4 and zDHHC5, two DHHCs we confirmed were targeted by CMA (Figure 4B, S11). The S-acylation of CD36 mediates its localization and in turn its fatty acid translocase activity ${ }^{49}$. We found that treatment of 3T3-L1 preadipocytes with CMA not only reduced CD36 acylation levels (Figure 5C), but also decreased the uptake of fatty acids and formation of lipid droplets, results previously observed with knockdown of zDHHC4/5 (Figure 5D, S19). Together, these results indicate that not only does CMA perturb the acylation of significant endogenous targets, it also modulates their known acylation-dependent activity and functionality. 


\section{Conclusions}

As our understanding of the role of protein S-acylation in regulating cell and organismal biology grows, the toxicity and limited potency of current inhibitors demand improved chemical tools. In this Article, we introduced CMA, a broad-spectrum inhibitor of DHHC family proteins, to complement current methods to probe $S$-acylation and its consequences in cells. Featuring an acrylamide warhead functionalized with a cyanomethyl group, CMA's potency is on par with 2BP - the leading inhibitor in the field - and, more importantly, has a significantly improved toxicity profile across multiple mammalian cells lines. We also show that CMA does not inhibit eraser APTs, a critical limitation of 2BP. These improvements were achieved without a loss of in-cell efficacy; for example, CMA inhibited the acylation of CD36 at $20 \mu \mathrm{M}$ under our conditions, while 2BP was used at $100 \mu \mathrm{M}^{49}$. Furthermore, we used 8, an alkyne-containing derivative of CMA, to show that CMA engages with DHHC family proteins across clades, validating CMA as a broadspectrum DHHC inhibitor and confirming its role in perturbing DHHC-dependent acylation. Finally, we applied CMA to assess cellular responses previously reported to result from loss of $S$ acylation. We demonstrated that decreases in the acylation of EGFR and CD36 caused by CMA treatment were paired with changes in signaling activity and lipid uptake, respectively - changes aligned with results reported using both genetic manipulation and 2BP treatment ${ }^{21,27}$. These results not only emphasize the importance of $S$-acylation in biological processes, but also highlight the potential of CMA in elucidating the regulatory roles of DHHC family proteins.

While this work reveals the promise of acrylamide based DHHC family inhibitors, it also underscores the need for additional improvements in selectivity and potency. MS analysis suggests that 8 can label 271 proteins with high confidence in cells, over $75 \%$ of which are annotated as acylated proteins (Table S1). These results suggest that 8 , and in turn CMA, interact with acylated targets and emphasize the disadvantage of their lipid "tails." As mimetics of fatty acids and their derivatives, aliphatic chains are thought to lead to numerous off-targets in cells ${ }^{50}$. Therefore, modification or replacement of the lipid will be critical in the next generation of molecules. As comparison of 1 with 2 indicates, though, the loss of hydrophobic contacts would need to be compensated for by expansion of the scaffold and increased contacts with the protein elsewhere. Interestingly, we observed that the cyanomethyl group was crucial for CMA potency; however, the reasons for its criticality are unclear. We envision that a crystal structure of a CMAbound DHHC would both aid in deciphering the mechanism of the cyanomethyl-mediated increase in potency and highlight opportunities for structure-based design. The ability of 10 to inhibit S-acylation in cellulo, but not zDHHC20 in vitro, was also intriguing and suggests that some 
members of our acrylamide panel might serve as a starting point for a DHHC inhibitor with intrafamily specificity. In addition, the capability of zDHHC20 to acylate NRas peptide, as well as recent reports of DHHC-targeted peptide inhibitors, hint at the potential of peptide scaffolds as DHHC inhibitors ${ }^{51-52}$. Our future work will focus on modifying small molecule and peptide scaffolds to achieve improvements in selectivity and potency.

\section{Acknowledgements}

This research was supported by the University of Chicago, the National Institute of General Medical Sciences of the National Institutes of Health NIH (R35 GM119840, to B.C.D.), the National Institute of Diabetes and Digestive and Kidney Diseases of the National Institutes of Health (F30 DK125088, to S-A.A.), and the Swiss National Science Foundation (P2BEP2_188250, to C.D.). We thank the Mass Spectrometry Core facility at University of Illinois at Chicago for providing technical advice on sample preparation for proteomics. We thank S. Bamji (University of British Columbia), M. Fukata (NIPS, Japan), M. Machner (NIH) and B. Beutler (University of Texas Southwestern Medical Center) for providing the various plasmids used in this work.

\section{Notes}

R.S.K. and B.C.D. have a patent on the DPP technology used in this work.

\section{Supporting Information}

Supporting figures, tables, spectra, procedures, and analysis 


\section{Methods}

In vitro fluorescence polarization assay of $\mathrm{ZDHHC}_{20}$ for molecule screening and $\mathrm{IC}_{50}$ determination with 1-hour preincubation

We used a $48 \mu \mathrm{l}$ protocol for initial molecule screening and $\mathrm{IC}_{50}$ determination with 1 hour of preincubation. Purified zDHHC20 (64 nM in $12 \mu \mathrm{L})$ in reaction buffer (50 mM HEPES, $150 \mathrm{mM}$ $\mathrm{NaCl}, 1 \mathrm{mM}$ EDTA, 2 mM TCEP, 2 mM DDM, pH 7.0) or reaction buffer with protein storage buffer was added to a 384-well optical bottom plate (ThermoFisher). $12 \mu \mathrm{L}$ of the small molecule stock in reaction buffer were added, resulting in a $24 \mu \mathrm{L}$ mixture of enzyme and small molecule, and the mixtures were incubated in $37^{\circ} \mathrm{C}$ for 1 hour. The MasterMix II $(24 \mu \mathrm{L})$, containing $8 \mu \mathrm{M}$ palmitoyl-CoA and $2.5 \mu \mathrm{M}$ peptide 01(5-FAM-GTQGCMGLPCVVM-COOH) in reaction buffer, was added to initiate the reaction, resulting in $4 \mu \mathrm{M}$ and $1.25 \mu \mathrm{M}$ as the final concentration of palmitoyl-CoA and peptide 01, respectively. Fluorescence measurements were recorded on Synergy Neo2 Hybrid Multi-Mode Reader (BioTek Instruments, Inc.) with Dual FP Green filter cube. Fluorescence polarization $\left(\lambda_{\mathrm{ex}}=485 / 20 \mathrm{~nm}, \lambda_{\mathrm{em}}=528 / 20 \mathrm{~nm}\right.$, gain=35, read from the top with height $7.5 \mathrm{~mm}$, and filter switching method) was measured at 1-min time intervals for 2 hours at $37^{\circ} \mathrm{C}$. Assay data were exported in Microsoft Excel 2016. For all of the molecule screening and $\mathrm{IC}_{50}$ determination, we performed the assay in kinetics mode, and data points $\mathrm{at} \mathrm{t}=118$ min were utilized as readout of enzyme activities of different samples for comparison.

\section{In vitro assay of APT inhibition}

Purified APT1 or APT2 (200 nM in $75 \mu \mathrm{L}$ ) in DPBS or DPBS alone was added to the 96-well optical bottom plate (ThermoFisher), followed by $75 \mu \mathrm{L}$ of 50,100 , or $200 \mu \mathrm{M}$ of 2BP or CMA in DPBS. Controls were incubated with an equal volume of DMSO. The mixtures were incubated in $37^{\circ} \mathrm{C}$ for 1 hour. At the end of pre-incubation, $150 \mu \mathrm{L}$ of $2 \mu \mathrm{M}$ DPP- 5 solution in DPBS was added, resulting in a final concentration of: 50 nM APT1/2; $1 \mu$ M DPP-5; 0, 12.5, 25, $50 \mu \mathrm{M}$ 2BP or CMA. Fluorescence measurements were recorded on Synergy Neo2 Hybrid Multi-Mode Reader (BioTek Instruments, Inc.). Fluorescence intensities (excitation wavelength $\lambda_{\mathrm{ex}}=490 / 20 \mathrm{~nm}$, emission wavelength $\lambda_{\mathrm{em}}=545 / 20 \mathrm{~nm}$, gain=80, read from bottom with height $4.5 \mathrm{~mm}$ and sweep method) were measured at 15 -second time intervals for 1 hour at $37^{\circ} \mathrm{C}$. The initial velocity was calculated from linear regression of the first 11 data points which was done using GraphPad Prism 8. 


\section{Acyl-biotin exchange (ABE) assay}

A $10 \mathrm{~cm}$ plate of cells was washed twice with cold DPBS, lysed with 1-2 mL of RIPA lysis buffer supplemented with a protease inhibitor cocktail and $50 \mathrm{mM} \mathrm{N}$-ethylmaleimide (NEM) (Acros), and subjected to end-over-end rotation at $4{ }^{\circ} \mathrm{C}$ for at least three hours. Following centrifugation at $13,000 \mathrm{~g}$ for $20 \mathrm{~min}$. at $4{ }^{\circ} \mathrm{C}$, the supernatant was collected. Protein concentration was measured using the BCA assay (ThermoFisher), and equal amounts of total protein from each sample were then subjected to acetone precipitation. The resulting pellet was dissolved by sonication in resuspension buffer (4\% SDS, 50 mM HEPES, 150 mM NaCl, 5 mM EDTA, pH 7.4; $100 \mu \mathrm{L}$ per $\mathrm{mg}$ of protein) containing $50 \mathrm{mM}$ NEM, and then Triton buffer ( $0.2 \%$ Triton X-100, $50 \mathrm{mM}$ HEPES, $150 \mathrm{mM} \mathrm{NaCl}, 5 \mathrm{mM}$ EDTA, pH 7.4; $250 \mu \mathrm{L}$ per mg of protein) containing $50 \mathrm{mM}$ NEM was added. This protein solution was rotated end-over-end for 2-3 hours at $25^{\circ} \mathrm{C}$, followed by two chloroformmethanol precipitations to remove excess NEM. The resulting protein pellet was dissolved in $80 \mu \mathrm{L}$ of resuspension buffer via sonication. The protein sample was divided into two equal parts for \pm hydroxylamine (HA) (Combi-Blocks) treatment. Each sample was treated with $160 \mu \mathrm{L}$ of either -HA buffer (Triton buffer) or +HA buffer (Triton buffer containing 0.7 M HA, pH 7.2-7.4). Samples were incubated for 1 hour at room temperature with shaking, and then proteins were precipitated by chloroform-methanol precipitation to remove excess HA. Protein pellets were resuspended by sonication in $40 \mu \mathrm{L}$ of resuspension buffer containing $10 \mu \mathrm{M}$ EZ-Link HPDP-Biotin (ThermoFisher), diluted with $160 \mu \mathrm{L}$ of Triton buffer containing $10 \mu \mathrm{M}$ HPDP-Biotin, and incubated for 2 hours at room temperature with shaking. Excess biotin was removed with two sequential chloroform-methanol precipitations. Protein pellets were dissolved in $40 \mu \mathrm{L}$ resuspension buffer by sonicating, and the solution volume was brought to $400 \mu \mathrm{L}$ with Triton buffer. Protein (25-25 $\mu \mathrm{g})$ was removed to serve as an expression control ('input'). The remaining solution was diluted with Triton buffer, and streptavidin-agarose beads (50 $\mu \mathrm{L}$ of slurry per $1 \mathrm{mg}$ of protein) were added to each sample, which were then incubated at $4{ }^{\circ} \mathrm{C}$ overnight with end-over-end rotation. Unbound proteins were removed by washing with $1 \mathrm{~mL}$ wash buffer $(0.1 \%$ SDS, $0.2 \%$ Triton X100, 50 mM HEPES, $150 \mathrm{mM} \mathrm{NaCl}, 5 \mathrm{mM}$ EDTA, pH 7.4). Bound proteins ('output') were eluted by boiling the beads for $10 \mathrm{~min}$ at $95^{\circ} \mathrm{C}$ with $1 \times$ Laemelli sample buffer (Alfa Aesar) containing $9 \%$ $\beta$-mercaptoethanol or $30 \mathrm{mM}$ DTT. The protein was resolved on $8-12 \%$ SDS-PAGE gels and subjected to Western blotting using the protocol described above.

\section{CMA ABE assay}

At $40-70 \%$ confluency, $10-\mathrm{cm}$ plates of HEK293T cells were transfected with $4 \mu \mathrm{g}$ pcDNA.6.1eGFP-GobX or $6 \mu \mathrm{g}$ pCMV-HA-Myd88 plasmid. At 24 hours post transfection, cells were treated 
with CMA $(0,5,10$, and $20 \mu \mathrm{M})$ for 6 hours. For endogenous targets, $10 \mathrm{~cm}$ dishes of MDA-MB231 or 3T3-L1 preadipocytes at 80\% confluency were treated with CMA $(0,20 \mu \mathrm{M})$ for 3 or 6 hours. Cells were then lysed and samples prepared in accordance with the procedure described in the ABE protocol.

\section{7-ODYA metabolic labeling assay}

HEK293T cells ( 200,000 cells per well) were plated in 12-well plate (Fisher) in growth medium. After 48 hours, cells were treated with various concentrations of CMA or 2BP in serum-free DMEM for 3 hours. After 1 or 3 hours of preincubation, the media was replaced with metabolic labeling media (serum-free DMEM with $50 \mathrm{mM}$ 17-ODYA preincubated with 5\% BSA) complemented with the same concentration of either CMA or 2BP, and the cells were then incubated for another 6 hours. Cells were lysed by SDS-free lysis buffer (50 mM HEPES, 150 mM NaCl, 1\% Triton-100, $0.5 \%$ sodium deoxycholate, $\mathrm{pH}=7.4$ ). If no hydroxylamine treatment, after centrifugation, the final cell lysate was collected and normalized to $1 \mathrm{mg} / \mathrm{ml}$ in $30 \mu \mathrm{L}$. For Cu-AAC conjugation, Master mix $(2.4 \mu \mathrm{L})$ made from equal volumes of $5 \mathrm{mM}$ TAMRA-azide (click chemistry tools), $5 \mathrm{mM}$ TBTA (Cayman), $50 \mathrm{mM} \mathrm{CuSO}_{4}$, and $50 \mathrm{mM}$ TCEP was added into $30 \mu \mathrm{L}$ cell lysate. The resulting solution was incubated for 1 hour at room temperature in darkness, then the reaction was quenched with $6 x$ Laemelli sample buffer containing $9 \% \beta$-mercaptoethanol before running the SDS-PAGE gels. For hydroxylamine treatment, after centrifugation, the final cell lysate was collected and normalized to $1 \mathrm{mg} / \mathrm{ml}$ in $100 \mu \mathrm{L}$. For Cu-AAC conjugation, Master mix $(8 \mu \mathrm{L})$ made from equal volumes of $5 \mathrm{mM}$ TAMRA-azide (click chemistry tools), $5 \mathrm{mM}$ TBTA (Cayman), $50 \mathrm{mM}$ $\mathrm{CuSO}_{4}$, and $50 \mathrm{mM}$ TCEP was added into $100 \mu \mathrm{L}$ cell lysate. The resulting solution was incubated for 1 hour at room temperature in darkness. The click reaction was quenched by chloroformmethanol precipitation. Each protein pellet was resuspended in resuspension buffer (the same in $A B E$ assay), then was divided into two equal parts for \pm hydroxylamine $(H A)$ treatment. Each sample was treated with $160 \mu \mathrm{L}$ of either -HA buffer (Triton buffer) or +HA buffer (Triton buffer containing 0.7 M HA, pH 7.2-7.4). Samples were incubated for 1 hour at room temperature with shaking, and then proteins were precipitated by chloroform-methanol precipitation to remove excess HA. Protein pellets were resuspended by sonication in $50 \mu \mathrm{L}$ of resuspension buffer and $6 x$ Laemelli sample buffer containing $9 \% \beta$-mercaptoethanol was added before running the gels. Proteins were resolved with $12 \%$ SDS-PAGE and visualized with FluoroChem R (Proteinsimple) using the MultiFluo-Green channel. Coomassie Blue was used to stain total protein. 


\section{MTS assay}

Cells ( 100,000 cells per well for HEK293T, 50,000 cells per well for 3T3-L1, MDA-MB-231, and HeLa, and $\sim 70,000$ cells per well for HepG2) were plated in 96-well plates (Corning) in growth media. After 24 hours, cells were washed once with DPBS and treated with CMA or 2BP in a concentration gradient for either 6 or 24 hours in serum-free DMEM (EMEM for HepG2 only). Then cells were washed by DPBS and then treated with the MTS reagent (Abcam) in serum-free DMEM GlutaMAX (EMEM, HepG2 only) for 2-3 hours. Absorption at $490 \mathrm{~nm}$ was measured on Synergy Neo2 Hybrid Multi-Mode Reader (BioTek Instruments, Inc.). The data are taken from at least $n=2$ independent experiments containing at least two replicates each time. Assay data were analyzed using Microsoft Excel 2016 and nonlinear curve fitting was performed using GraphPad Prism 8.

\section{In cellulo labeling assay of DHHC library}

HEK293T cells ( $~ 82,500$ cells per well) were plated in 24-well plate (Fisher). For human DHHC labeling, after 20-24 hours, cells were transfected with $0.6 \mu \mathrm{g}$ plasmids with PEI. For mouse DHHC labeling, cells were transfected with $0.6 \mu \mathrm{g}$ plasmids with Lipofectamine 3000 (ThermoFisher) according to manufacturer's protocol. For 8 labeling assay, at 24 hours post transfection, cells were incubated with serum-free DMEM complemented with $\mathbf{8}$ for 2 hours. For CMA competitive labeling assay, at 24 hours post transfection, the media was removed, and cells were incubated with serum-free DMEM with CMA for 2 hours, then 8 in DMEM was added into media, resulting in a final concentration of 8 at $1 \mu \mathrm{M}$. Cells then were washed with DPBS twice and lysed with $50 \mu \mathrm{L}$ RIPA buffer (50 mM HEPES, $150 \mathrm{mM} \mathrm{NaCl}, 1 \%$ Triton-100, 0.5\% sodium deoxycholate, $0.1 \% \mathrm{SDS}, \mathrm{pH}=7.4$ ). After spun down, the final cell lysate was collected and normalized to $1 \mathrm{mg} / \mathrm{mL}$ in $30 \mu \mathrm{L}$ before $\mathrm{Cu}-\mathrm{AAC}$ conjugation. Cu-AAC conjugation with TAMRAazide was performed according to the protocol shown above. Proteins were resolved with 12\% SDS-PAGE and visualized with FluoroChem R (Proteinsimple) using the MultiFluo-Green channel. Western blots of anti-myc or anti-HA were used as a control to indicate the molecular sizes of $\mathrm{DHHC}$ proteins and the amount of DHHC proteins. Cells were labelled in serum-free media without further notice. When cells were labelled in serum-complemented media $(0.1 \%$ or $1 \%$ FBScomplemented DMEM), for 8 labeling assay, at 24 hours post transfection, cells were incubated with DMEM complemented with 8 and corresponding concentration of FBS for 2 hours. For CMA competitive labeling assay, at 24 hours post transfection, the media was removed, and cells were 
incubated with DMEM complemented with CMA and corresponding concentration of FBS for 2 hours, then 8 in DMEM was added into media, resulting in a final concentration of 8 at $10 \mu \mathrm{M}$.

\section{In cellulo labeling of endogenous DHHC proteins}

At $80-90 \%$ confluency, $10 \mathrm{~cm}$ dishes of HEK293T cells were incubated with DMSO or $20 \mu \mathrm{M}$ CMA in serum-free DMEM GlutaMAX for 3 hours, followed DMSO or $20 \mu \mathrm{M} 8$ for an additional 3 hours. After treatment, cells were washed twice with cold DPBS and lysed in $1 \mathrm{~mL}$ of SDS-free RIPA buffer for 1 hour at $4{ }^{\circ} \mathrm{C}$. Following centrifugation at $13,000 \mathrm{~g}$ for $20 \mathrm{~min}$. at $4{ }^{\circ} \mathrm{C}$, the supernatant was collected, and protein concentration was determined using a BCA assay. Samples were normalized to $1 \mathrm{mg} / \mathrm{mL}$. For Cu-AAC conjugation, a master mix (equal volumes of $5 \mathrm{mM}$ biotinazide (Click Chemistry Tools), $5 \mathrm{mM}$ TBTA (Cayman), $50 \mathrm{mM} \mathrm{CuSO}_{4}$, and $5 \mathrm{mM}$ TCEP) was prepared and added to each sample (16 $\mu \mathrm{L}$ for $184 \mu \mathrm{g}$ protein). The resulting solution was rotated for 1 hour at room temperature and then quenched with $100 \mathrm{mM}$ EDTA (100x) and subjected to two chloroform-methanol precipitations. Protein pellets were dissolved in $40 \mu \mathrm{L} A B E$ resuspension buffer by sonicating, and the total volume was brought to $400 \mu \mathrm{L}$ with ABE Triton buffer. Protein pellets were dissolved in $40 \mu \mathrm{L}$ resuspension buffer by sonicating, and the solution volume was brought to $400 \mu \mathrm{L}$ with Triton buffer. Protein (20-25 $\mu \mathrm{g})$ was removed to serve as an expression control ('input'). The remaining solution was diluted with Triton buffer, and streptavidin-agarose beads ( $50 \mu \mathrm{L}$ of slurry per $1 \mathrm{mg}$ of protein) were added to each sample, which were then incubated at $4{ }^{\circ} \mathrm{C}$ overnight with end-over-end rotation. Unbound proteins were removed by washing three times with $1 \mathrm{ml}$ of $A B E$ wash buffer. Bound proteins ('output') were eluted by boiling the beads for $10 \mathrm{~min}$ at $95^{\circ} \mathrm{C}$ with $1 \mathrm{x}$ Laemelli sample buffer (Alfa Aesar) containing 20-30 mM DTT. The protein was resolved on 8-12\% SDS-PAGE gels and subjected to Western blotting using the protocol described above. For non-competitive 8 labeling, the CMA pretreatment step was not followed.

\section{Lipid uptake assays}

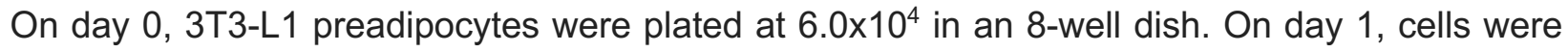
cultured in serum-free media for 12 hours, and then, on day 2, treated with either CMA (20 uM) or DMSO for 3 hours, followed by treatment with $10 \mu \mathrm{M}$ BSA-bound oleate and $2 \mu \mathrm{M}$ BODIPY 493/503 or BODIPY-palmitate for 6 hours. DAPI was then added, and then cells were washed and fixed with $4 \%$ paraformaldehyde. Cells were washed $(5 \times 5 \mathrm{~min}$.) with PBS and mounted in Prolong Antifade Mountant (Thermo Fisher). Slides were imaged using an inverted epifluoresence microscope (Leica DMi8) equipped with a Hamamatsu Orca-Flash 4.0 camera, a 63x oil objective 
(numerical aperture 1.4), and a $300 \mathrm{~W}$ Xenon light source (Sutter Lamba XL). LASX software was used to obtain BODIPY and brightfield images. 


\section{Figure Legends}

A

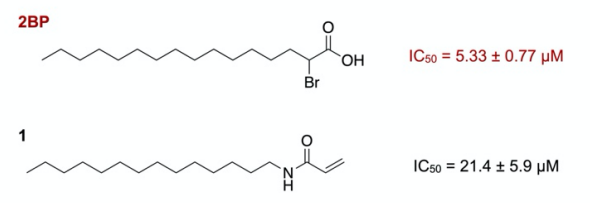

CMA

$\mathrm{IC}_{50}=1.35 \pm 0.26 \mu \mathrm{M}$

8

$\mathrm{IC}_{50}=8.32 \pm 2.25 \mu \mathrm{M}$

B

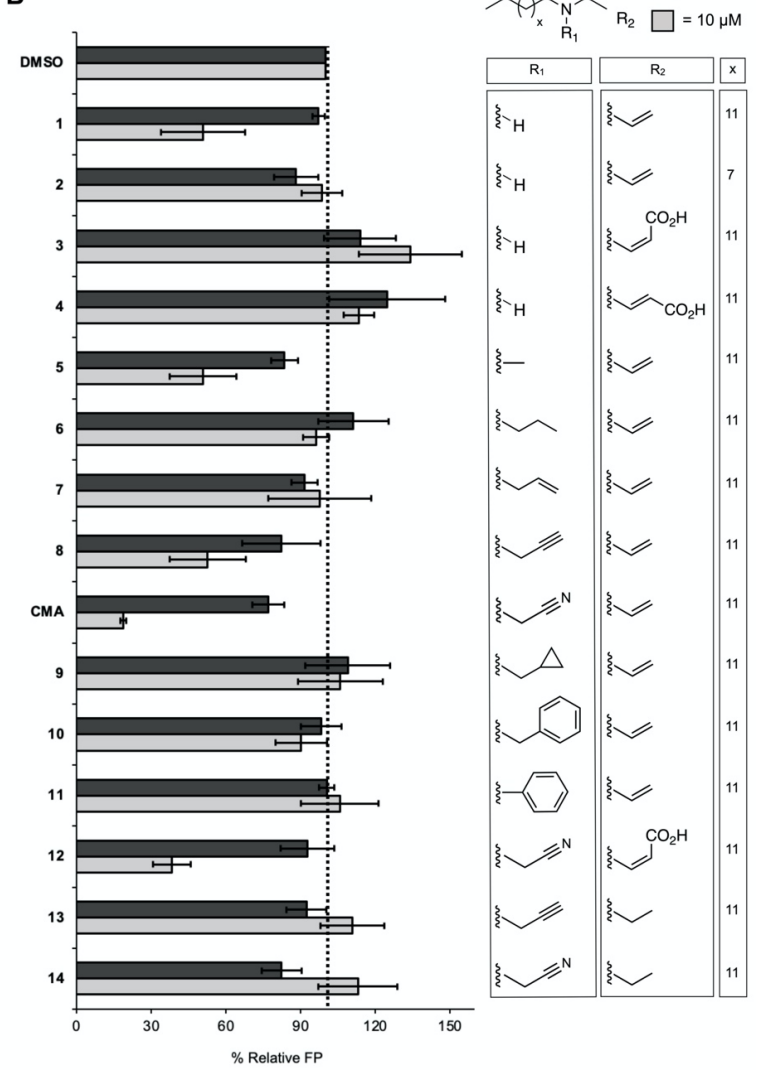

Figure 1. Discovery of and in vitro inhibition data against human zDHHC20 for CMA. (A) Structure and $\mathrm{IC}_{50}$ against human $\mathrm{zDHHC20}$ of key molecules used in this work. (B) Fluorescence polarization (FP) screening of a panel of acrylamide-based molecules against zDHHC20, with activity normalized to DMSO. Data are presented as the mean \pm standard deviation $(n=3)$. 
A

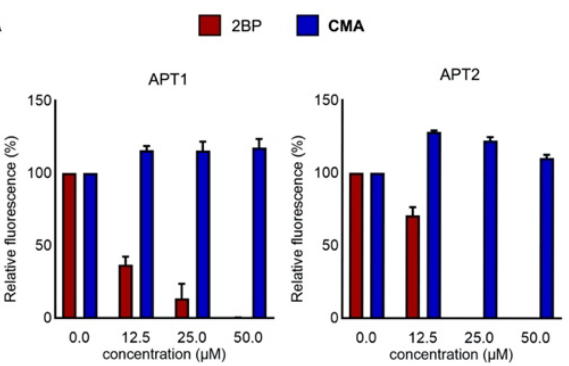

B

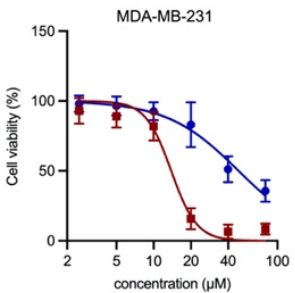

$\square$ 2BP $\square$ CMA

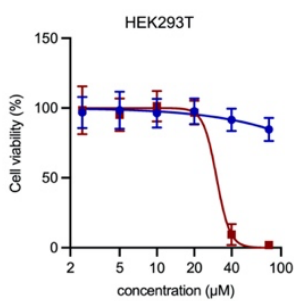

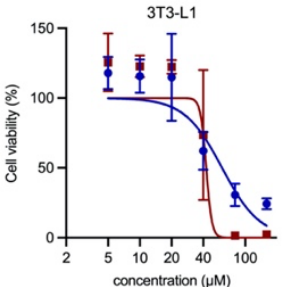

Figure 2. Characterization of CMA (A) Incubation of purified APT1 or APT2 (50 nM) with either 2BP or CMA, followed by measurement of APT activity using the fluorogenic probe DPP-5. Data are presented as the mean \pm standard deviation $(n=3)$. (B) Viability of HEK293T, MDA-MB-231, and 3T3-L1 cells treated with varied concentrations of either 2BP or CMA (24 hours), as measured by MTS assay. Data are presented as the mean \pm standard deviation $(n \geq 3)$. 


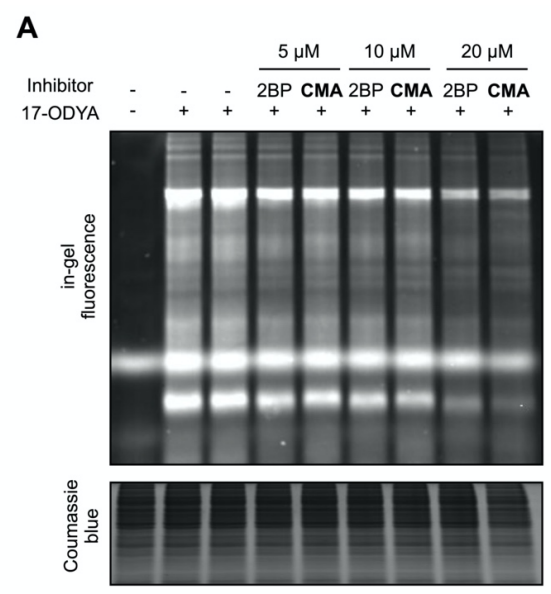

B

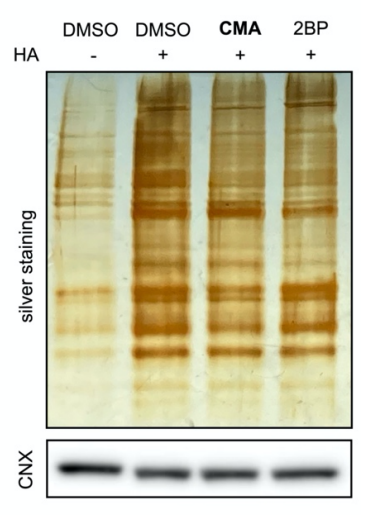

C
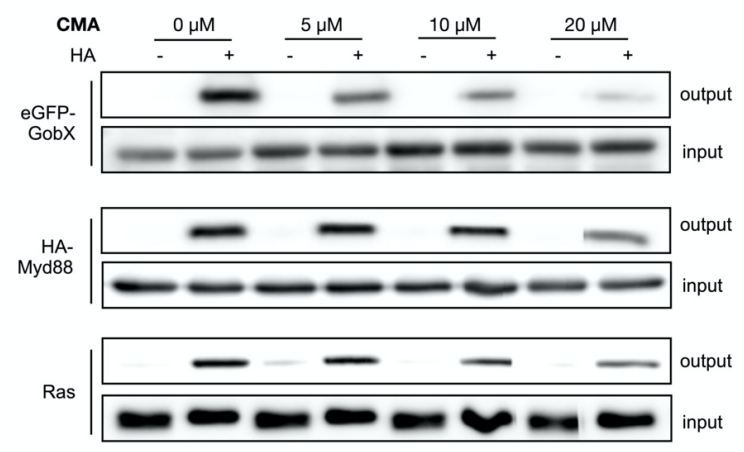

D

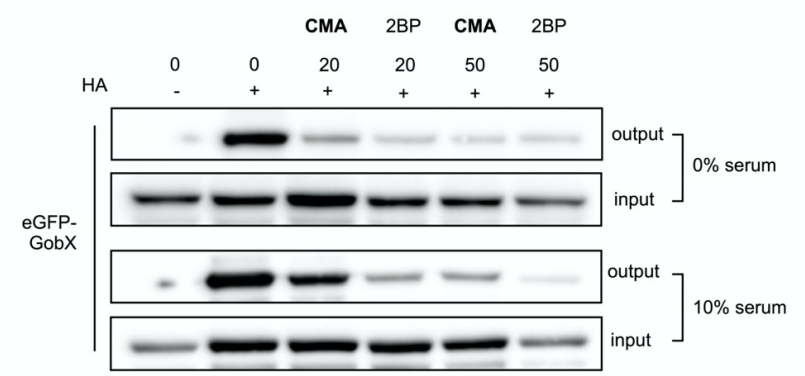

Figure 3. Validation of CMA in cellulo (A) HEK293T cells pretreated with DMSO, 2BP or CMA (0, 5,10 , and $20 \mu \mathrm{M}, 3$ hours) before treatment with 17-octadecynoic acid (17-ODYA) (6 hours) to metabolically label palmitoylated proteins. Isolated proteomes were then subject to click chemistry to conjugate TAMRA-azide to proteins modified by 17-ODYA, followed by protein separation via SDS-PAGE. In-gel fluorescence revealed a proteome-wide decrease in protein palmitoylation with CMA treatment. (B) Acyl-biotin exchange (ABE) of HEK293T cells treated with 2BP or CMA $(20 \mu \mathrm{M}, 6$ hours). Global S-acylation visualized using silver staining, with $\mathrm{CNX}$ as a loading control $(n \geq 2)$. (C) Dose-response change in the S-acylation of exogenous eGFP-tagged GobX or HAtagged Myd88 and endogenous Ras in HEK293T cells upon CMA treatment (6 hours) as measured by $A B E$, carried out in serum-free media. CNX serves as an assay and loading control $(n \geq 2)$. (D) Acyl-biotin exchange (ABE) of HEK293T cells expressing eGFP-tagged GobX and treated with $2 \mathrm{BP}$ or CMA $(0,20,50 \mu \mathrm{M})$ carried out in serum-free or serum-full media. ' 
A
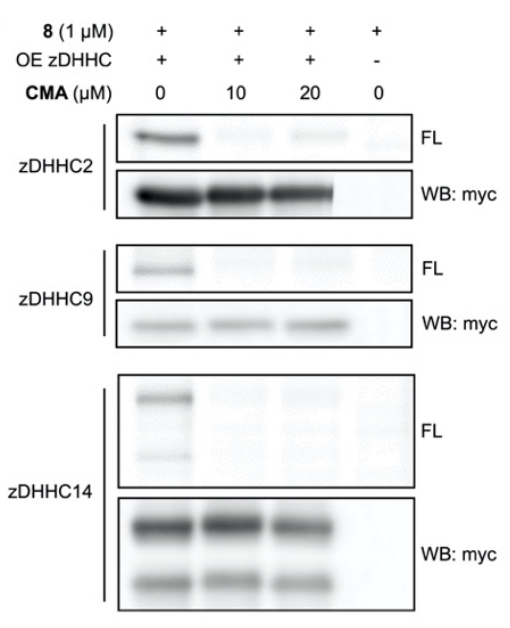
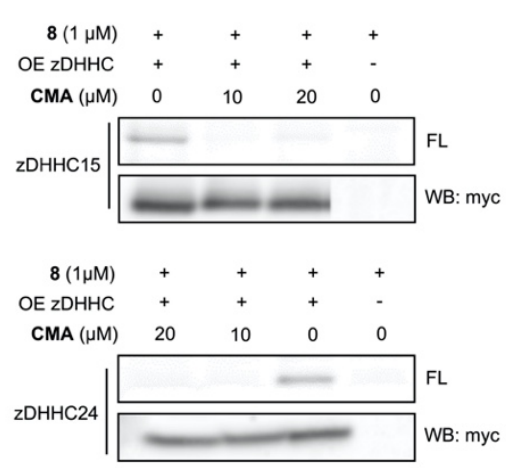

B

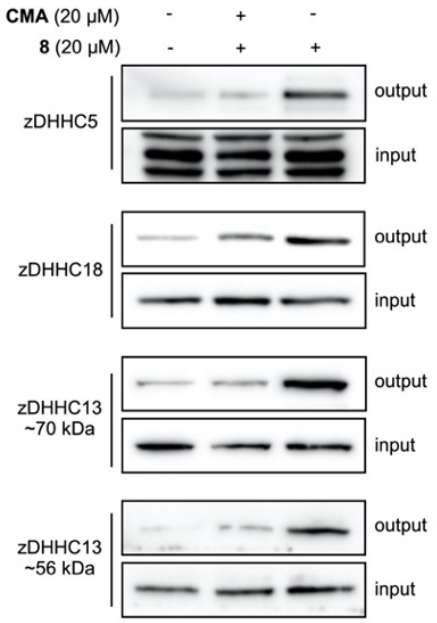

Figure 4. Engagement of CMA with DHHC family proteins. (A) In-gel fluorescence analysis of myc-tagged DHHC proteins overexpressed in HEK293T cells treated with CMA or DMSO (2 hours), followed by labeling with 8 ( $1 \mu \mathrm{M}, 2$ hours). Cell lysates subject to click chemistry to conjugate TAMRA-azide to proteins modified by $\mathbf{8}$, followed by protein separation via SDS-PAGE. Expression levels visualized via anti-myc tag Western blot $(n \geq 2)$. (B) Western blot analysis of endogenous DHHC proteins in HEK293T cells treated with CMA or DMSO (3 hours), followed by labeling with 8 ( $20 \mu \mathrm{M}, 3$ hours). Cell lysates were subject to click chemistry to conjugate biotinazide to proteins modified by $\mathbf{8}$, followed by streptavidin enrichment, protein separation via SDSPAGE, and Western blotting for indicated targets $(n \geq 2)$. 
A

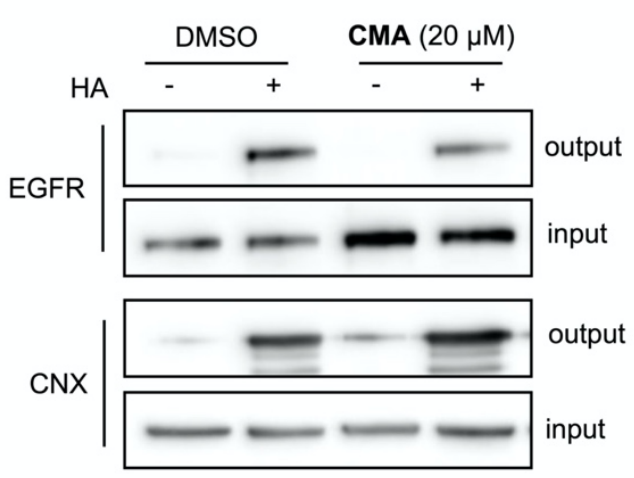

C
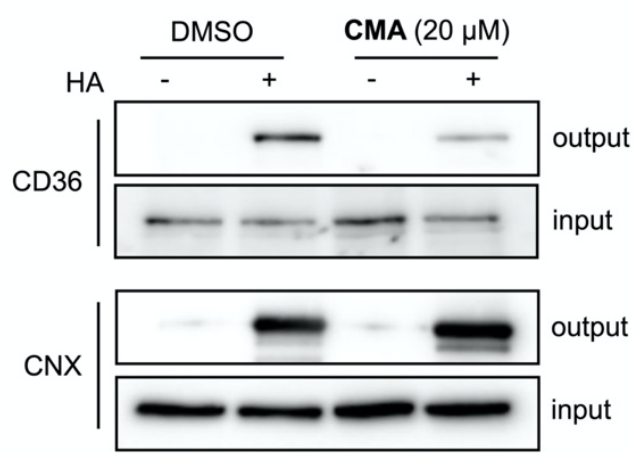

B

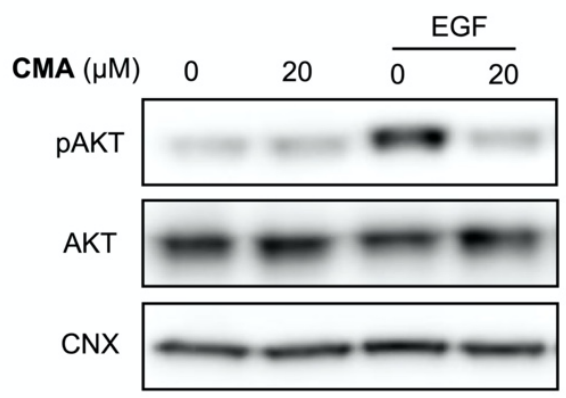

D

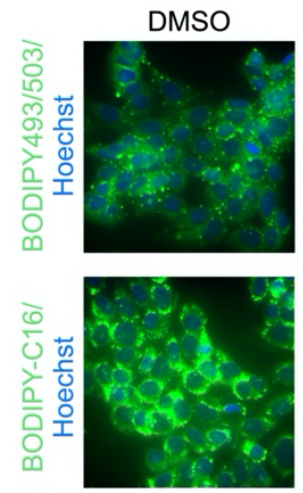

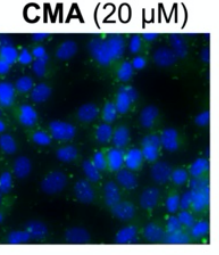

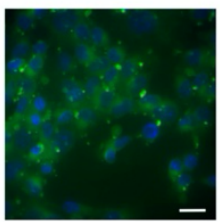

NIH 3T3-L1s

Figure 5. Confirmation of CMA functional activity in cellul o. (A) Analysis of EGFR S-acylation via ABE upon CMA treatment (20 $\mu \mathrm{M}, 3$ hours) in MDA-MB-231 cells, with CNX as an assay and loading control $(n \geq 2)$. (B) EGF (100 $\mathrm{ng} / \mathrm{mL}, 15$ minutes)-induced phosphorylation of AKT with and without CMA treatment $(20 \mu \mathrm{M}, 3$ hours $)(n \geq 2)$. (C) Analysis of CD36 S-acylation via ABE upon CMA treatment $(20 \mu \mathrm{M}, 6$ hours $)$ in 3T3-L1 cells, with CNX as an assay and loading control $(n \geq 2)$. (D) Representative images of fluorescence imaging of 3T3-L1 preadipocytes starved (12 hours) and treated with DMSO or CMA (20 $\mu \mathrm{M}, 3$ hours), followed by $10 \mu \mathrm{M}$ BSA-bound oleate and BODIPY493/503 or BODIPY-palmitate $(2 \mu \mathrm{M}, 3$ hours $)(n=5)$. Scale bar $=20 \mu \mathrm{M}$. 


\section{References}

1. Blanc, M.; David, F.; Abrami, L.; Migliozzi, D.; Armand, F.; Burgi, J.; van der Goot, F. G., SwissPalm: Protein Palmitoylation database. F1000Res 2015, 4, 261.

2. Lanyon-Hogg, T.; Faronato, M.; Serwa, R. A.; Tate, E. W., Dynamic Protein Acylation: New Substrates, Mechanisms, and Drug Targets. Trends Biochem Sci 2017, 42 (7), 566-581.

3. Linder, M. E.; Deschenes, R. J., Palmitoylation: policing protein stability and traffic. Nat Rev Mol Cell Biol 2007, 8 (1), 74-84.

4. Bhattacharyya, R.; Fenn, R. H.; Barren, C.; Tanzi, R. E.; Kovacs, D. M., Palmitoylated APP Forms Dimers, Cleaved by BACE1. PLoS One 2016, 11 (11), e0166400.

5. Seno, K.; Hayashi, F., Palmitoylation is a prerequisite for dimerization-dependent raftophilicity of rhodopsin. J Biol Chem 2017, 292 (37), 15321-15328.

6. Cao, Y.; Qiu, T.; Kathayat, R. S.; Azizi, S. A.; Thorne, A. K.; Ahn, D.; Fukata, Y.; Fukata, M.; Rice, P. A.; Dickinson, B. C., ABHD10 is an S-depalmitoylase affecting redox homeostasis through peroxiredoxin-5. Nat Chem Biol 2019, 15 (12), 1232-1240.

7. Bolland, D. E.; Moritz, A. E.; Stanislowski, D. J.; Vaughan, R. A.; Foster, J. D., Palmitoylation by Multiple DHHC Enzymes Enhances Dopamine Transporter Function and Stability. ACS Chem Neurosci 2019, 10 (6), 2707-2717.

8. Duncan, J. A.; Gilman, A. G., A cytoplasmic acyl-protein thioesterase that removes palmitate from G protein alpha subunits and p21(RAS). J Biol Chem 1998, 273 (25), 15830-7.

9. Tomatis, V. M.; Trenchi, A.; Gomez, G. A.; Daniotti, J. L., Acyl-protein thioesterase 2 catalyzes the deacylation of peripheral membrane-associated GAP-43. PLOS One 2010, 5 (11), e15045.

10. Lin, D. T.; Conibear, E., ABHD17 proteins are novel protein depalmitoylases that regulate N-Ras palmitate turnover and subcellular localization. Elife 2015, 4, e11306.

11. Ladygina, N.; Martin, B. R.; Altman, A., Dynamic palmitoylation and the role of DHHC proteins in T cell activation and anergy. Adv Immunol 2011, 109, 1-44.

12. Jiang, H.; Zhang, X.; Chen, X.; Aramsangtienchai, P.; Tong, Z.; Lin, H., Protein Lipidation: Occurrence, Mechanisms, Biological Functions, and Enabling Technologies. Chem Rev 2018, 118 (3), 919-988.

13. Gok, C.; Fuller, W., Topical review: Shedding light on molecular and cellular consequences of NCX1 palmitoylation. Cell Signal 2020, 76, 109791.

14. Chen, X.; Ma, H.; Wang, Z.; Zhang, S.; Yang, H.; Fang, Z., EZH2 Palmitoylation Mediated by ZDHHC5 in p53-Mutant Glioma Drives Malignant Development and Progression. Cancer Res 2017, 77 (18), 4998-5010.

15. Beard, R. S., Jr.; Yang, X.; Meegan, J. E.; Overstreet, J. W.; Yang, C. G.; Elliott, J. A.; Reynolds, J. J.; Cha, B. J.; Pivetti, C. D.; Mitchell, D. A.; Wu, M. H.; Deschenes, R. J.; Yuan, S. Y., Palmitoyl acyltransferase DHHC21 mediates endothelial dysfunction in systemic inflammatory response syndrome. Nat Commun 2016, 7, 12823.

16. Mukai, J.; Liu, H.; Burt, R. A.; Swor, D. E.; Lai, W. S.; Karayiorgou, M.; Gogos, J. A., Evidence that the gene encoding ZDHHC8 contributes to the risk of schizophrenia. Nat Genet 2004, 36 (7), 725-31.

17. Young, E.; Zheng, Z. Y.; Wilkins, A. D.; Jeong, H. T.; Li, M.; Lichtarge, O.; Chang, E. C., Regulation of Ras localization and cell transformation by evolutionarily conserved palmitoyltransferases. Mol Cell Biol 2014, 34 (3), 374-85.

18. Liu, P.; Jiao, B.; Zhang, R.; Zhao, H.; Zhang, C.; Wu, M.; Li, D.; Zhao, X.; Quu, Q.; Li, J.; Ren, R., Palmitoylacyltransferase Zdhhc9 inactivation mitigates leukemogenic potential of oncogenic Nras. Leukemia 2016, 30 (5), 1225-8.

19. Raymond, F. L.; Tarpey, P. S.; Edkins, S.; Tofts, C.; O'Meara, S.; Teague, J.; Butler, A.; Stevens, C.; Barthorpe, S.; Buck, G.; Cole, J.; Dicks, E.; Gray, K.; Halliday, K.; Hills, K.; Hinton, J.; Jones, D.; Menzies, A.; Perry, J.; Raine, K.; Shepherd, R.; Small, A.; Varian, J.; Widaa, S.; Mallya, U.; Moon, J.; Luo, Y.; Shaw, M.; Boyle, J.; Kerr, B.; Turner, G.; Quarrell, O.; Cole, T.; Easton, D. F.; Wooster, R.; Bobrow, M.; Schwartz, C. E.; Gecz, J.; Stratton, M. R.; Futreal, P. A., Mutations in ZDHHC9, which encodes a palmitoyltransferase of NRAS and HRAS, cause X-linked mental retardation associated with a Marfanoid habitus. Am J Hum Genet 2007, 80 (5), 982-7. 
20. Tarpey, P. S.; Smith, R.; Pleasance, E.; Whibley, A.; Edkins, S.; Hardy, C.; O'Meara, S.; Latimer, C.; Dicks, E.; Menzies, A.; Stephens, P.; Blow, M.; Greenman, C.; Xue, Y.; Tyler-Smith, C.; Thompson, D.; Gray, K.; Andrews, J.; Barthorpe, S.; Buck, G.; Cole, J.; Dunmore, R.; Jones, D.; Maddison, M.; Mironenko, T.; Turner, R.; Turrell, K.; Varian, J.; West, S.; Widaa, S.; Wray, P.; Teague, J.; Butler, A.; Jenkinson, A.; Jia, M.; Richardson, D.; Shepherd, R.; Wooster, R.; Tejada, M. I.; Martinez, F.; Carvill, G.; Goliath, R.; de Brouwer, A. P.; van Bokhoven, H.; Van Esch, H.; Chelly, J.; Raynaud, M.; Ropers, H. H.; Abidi, F. E.; Srivastava, A. K.; Cox, J.; Luo, Y.; Mallya, U.; Moon, J.; Parnau, J.; Mohammed, S.; Tolmie, J. L.; Shoubridge, C.; Corbett, M.; Gardner, A.; Haan, E.; Rujirabanjerd, S.; Shaw, M.; Vandeleur, L.; Fullston, T.; Easton, D. F.; Boyle, J.; Partington, M.; Hackett, A.; Field, M.; Skinner, C.; Stevenson, R. E.; Bobrow, M.; Turner, G.; Schwartz, C. E.; Gecz, J.; Raymond, F. L.; Futreal, P. A.; Stratton, M. R., A systematic, large-scale resequencing screen of X-chromosome coding exons in mental retardation. Nat Genet 2009, 41 (5), 535-43.

21. Runkle, K. B.; Kharbanda, A.; Stypulkowski, E.; Cao, X. J.; Wang, W.; Garcia, B. A.; Witze, E. S., Inhibition of DHHC20-Mediated EGFR Palmitoylation Creates a Dependence on EGFR Signaling. Mol Cell 2016, 62 (3), 385-396.

22. Kharbanda, A.; Walter, D. M.; Gudiel, A. A.; Schek, N.; Feldser, D. M.; Witze, E. S., Blocking EGFR palmitoylation suppresses PI3K signaling and mutant KRAS lung tumorigenesis. Sci Signal 2020, 13 (621).

23. Sanders, S. S.; Hayden, M. R., Aberrant palmitoylation in Huntington disease. Biochem Soc Trans 2015, 43 (2), 205-10.

24. Roth, A. F.; Feng, Y.; Chen, L.; Davis, N. G., The yeast DHHC cysteine-rich domain protein Akr1p is a palmitoyl transferase. J Cell Biol 2002, 159 (1), 23-8.

25. Lobo, S.; Greentree, W. K.; Linder, M. E.; Deschenes, R. J., Identification of a Ras palmitoyltransferase in Saccharomyces cerevisiae. J Biol Chem 2002, 277 (43), 41268-73.

26. Fukata, M.; Fukata, Y.; Adesnik, H.; Nicoll, R. A.; Bredt, D. S., Identification of PSD-95 palmitoylating enzymes. Neuron 2004, 44 (6), 987-96.

27. Wang, J.; Hao, J. W.; Wang, X.; Guo, H.; Sun, H. H.; Lai, X. Y.; Liu, L. Y.; Zhu, M.; Wang, H. Y.; Li, Y. F.; Yu, L. Y.; Xie, C.; Wang, H. R.; Mo, W.; Zhou, H. M.; Chen, S.; Liang, G.; Zhao, T. J., DHHC4 and DHHC5 Facilitate Fatty Acid Uptake by Palmitoylating and Targeting CD36 to the Plasma Membrane. Cell Rep 2019, 26 (1), 209-221 e5.

28. Abrami, L.; Dallavilla, T.; Sandoz, P. A.; Demir, M.; Kunz, B.; Savoglidis, G.; Hatzimanikatis, V.; van der Goot, F. G., Identification and dynamics of the human ZDHHC16-ZDHHC6 palmitoylation cascade. Elife 2017, 6.

29. Davda, D.; El Azzouny, M. A.; Tom, C. T.; Hernandez, J. L.; Majmudar, J. D.; Kennedy, R. T.; Martin, B. R., Profiling targets of the irreversible palmitoylation inhibitor 2-bromopalmitate. ACS Chem Biol 2013, 8 (9), 1912-7.

30. Pedro, M. P.; Vilcaes, A. A.; Tomatis, V. M.; Oliveira, R. G.; Gomez, G. A.; Daniotti, J. L., 2Bromopalmitate reduces protein deacylation by inhibition of acyl-protein thioesterase enzymatic activities. PLoS One 2013, 8 (10), e75232.

31. Tian, L.; McClafferty, H.; Jeffries, O.; Shipston, M. J., Multiple palmitoyltransferases are required for palmitoylation-dependent regulation of large conductance calcium- and voltage-activated potassium channels. J Biol Chem 2010, 285 (31), 23954-62.

32. Zheng, B.; Zhu, S.; Wu, X., Clickable analogue of cerulenin as chemical probe to explore protein palmitoylation. ACS Chem Biol 2015, 10 (1), 115-21.

33. DeJesus, G.; Bizzozero, O. A., Effect of 2-fluoropalmitate, cerulenin and tunicamycin on the palmitoylation and intracellular translocation of myelin proteolipid protein. Neurochem Res 2002, 27 (12), 1669-75.

34. Jennings, B. C.; Nadolski, M. J.; Ling, Y.; Baker, M. B.; Harrison, M. L.; Deschenes, R. J.; Linder, M. E., 2-Bromopalmitate and 2-(2-hydroxy-5-nitro-benzylidene)-benzo[b]thiophen-3-one inhibit DHHCmediated palmitoylation in vitro. $J$ Lipid Res 2009, 50 (2), 233-42.

35. Jackson, P. A.; Widen, J. C.; Harki, D. A.; Brummond, K. M., Covalent Modifiers: A Chemical Perspective on the Reactivity of alpha,beta-Unsaturated Carbonyls with Thiols via Hetero-Michael Addition Reactions. J Med Chem 2017, 60 (3), 839-885. 
36. Lanyon-Hogg, T.; Ritzefeld, M.; Sefer, L.; Bickel, J. K.; Rudolf, A. F.; Panyain, N.; Bineva-Todd, G.; Ocasio, C. A.; O'Reilly, N.; Siebold, C.; Magee, A. I.; Tate, E. W., Acylation-coupled lipophilic induction of polarisation (Acyl-cLIP): a universal assay for lipid transferase and hydrolase enzymes. Chem Sci 2019, 10 (39), 8995-9000.

37. Qiu, T.; Kathayat, R. S.; Cao, Y.; Beck, M. W.; Dickinson, B. C., A Fluorescent Probe with Improved Water Solubility Permits the Analysis of Protein S-Depalmitoylation Activity in Live Cells. Biochemistry 2018, 57 (2), 221-225.

38. Martin, B. R.; Cravatt, B. F., Large-scale profiling of protein palmitoylation in mammalian cells. Nat Methods 2009, 6 (2), 135-8.

39. Drisdel, R. C.; Green, W. N., Labeling and quantifying sites of protein palmitoylation. Biotechniques 2004, 36 (2), 276-85.

40. Kim, Y. C.; Lee, S. E.; Kim, S. K.; Jang, H. D.; Hwang, I.; Jin, S.; Hong, E. B.; Jang, K. S.; Kim, H. S., Toll-like receptor mediated inflammation requires FASN-dependent MYD88 palmitoylation. Nat Chem Biol 2019, 15 (9), 907-916.

41. Lin, Y. H.; Doms, A. G.; Cheng, E.; Kim, B.; Evans, T. R.; Machner, M. P., Host Cell-catalyzed SPalmitoylation Mediates Golgi Targeting of the Legionella Ubiquitin Ligase GobX. J Biol Chem 2015, 290 (42), 25766-81.

42. Dallavilla, T.; Abrami, L.; Sandoz, P. A.; Savoglidis, G.; Hatzimanikatis, V.; van der Goot, F. G., ModelDriven Understanding of Palmitoylation Dynamics: Regulated Acylation of the Endoplasmic Reticulum Chaperone Calnexin. PLoS Comput Biol 2016, 12 (2), e1004774.

43. Martin, B. R., Chemical approaches for profiling dynamic palmitoylation. Biochem Soc Trans 2013, 41 (1), 43-9.

44. Jennings, B. C.; Linder, M. E., DHHC protein S-acyltransferases use similar ping-pong kinetic mechanisms but display different acyl-CoA specificities. J Biol Chem 2012, 287 (10), 7236-45.

45. Mitchell, D. A.; Mitchell, G.; Ling, Y.; Budde, C.; Deschenes, R. J., Mutational analysis of Saccharomyces cerevisiae Erf2 reveals a two-step reaction mechanism for protein palmitoylation by DHHC enzymes. J Biol Chem 2010, 285 (49), 38104-14.

46. Zheng, B.; DeRan, M.; Li, X.; Liao, X.; Fukata, M.; Wu, X., 2-Bromopalmitate analogues as activitybased probes to explore palmitoyl acyltransferases. J Am Chem Soc 2013, 135 (19), 7082-5.

47. Bastin, G.; Dissanayake, K.; Langburt, D.; Tam, A. L. C.; Lee, S. H.; Lachhar, K.; Heximer, S. P., RGS4 controls Galphai3-mediated regulation of Bcl-2 phosphorylation on TGN38-containing intracellular membranes. J Cell Sci 2020, 133 (12).

48. Zhang, Y.; Lin, Z.; Tan, Y.; Bu, F.; Hao, P.; Zhang, K.; Yang, H.; Liu, S.; Ren, Y., Exploration of Missing Proteins by a Combination Approach to Enrich the Low-Abundance Hydrophobic Proteins from Four Cancer Cell Lines. J Proteome Res 2020, 19 (1), 401-408.

49. Hao, J. W.; Wang, J.; Guo, H.; Zhao, Y. Y.; Sun, H. H.; Li, Y. F.; Lai, X. Y.; Zhao, N.; Wang, X.; Xie, C.; Hong, L.; Huang, X.; Wang, H. R.; Li, C. B.; Liang, B.; Chen, S.; Zhao, T. J., CD36 facilitates fatty acid uptake by dynamic palmitoylation-regulated endocytosis. Nat Commun 2020, 11 (1), 4765.

50. Arnott, J. A.; Planey, S. L., The influence of lipophilicity in drug discovery and design. Expert Opin Drug Discov 2012, 7 (10), 863-75.

51. Yao, H.; Lan, J.; Li, C.; Shi, H.; Brosseau, J. P.; Wang, H.; Lu, H.; Fang, C.; Zhang, Y.; Liang, L.; Zhou, X.; Wang, C.; Xue, Y.; Cui, Y.; Xu, J., Inhibiting PD-L1 palmitoylation enhances T-cell immune responses against tumours. Nat Biomed Eng 2019, 3 (4), 306-317.

52. Yao, H.; Li, C.; He, F.; Song, T.; Brosseau, J.-P.; Wang, H.; Lu, H.; Fang, C.; Shi, H.; Lan, J.; Fang, J.-Y.; Xu, J., A peptidic inhibitor for PD-1 palmitoylation targets its expression and functions. RSC Chemical Biology 2021. 